\title{
Dinâmica do fósforo em ovinos alimentados com dietas contendo diversos níveis deste mineral ${ }^{1}$
}

\author{
Everton do Espírito Santo Borges ${ }^{2}$, José Cleto da Silva Filho ${ }^{3}$, Natália Charleaux Roque ${ }^{4}$, Juan \\ Ramón Olalquiaga Pérez ${ }^{3}$, Dorinha Miriam Silber Schmidt Vitti ${ }^{5}$, René Maurício Patiño Pardo ${ }^{6}$
}

${ }^{1}$ Projeto financiado pela FAPEMIG e Bolsa financiada pelo CNPq.

2 Mestrando do Programa de Pós-graduação em Zootecnia - UFLA/Lavras. Bolsista do CNPq.

${ }^{3}$ Departamento de Zootecnia, UFLA/Lavras.

${ }^{4}$ Curso de Graduação em Zootecnia/UFLA, Lavras, MG.

${ }^{5}$ Centro de Energia Nuclear na Agricultura (CENA), Universidade de São Paulo (USP), Piracicaba, SP.

${ }^{6}$ Faculdade de Ciências Agropecuárias (Universidad de Sucre - Colombia).

RESUMO - Avaliou-se a dinâmica do fósforo no organismo de cordeiros Santa Inês alimentados com dietas com diversos níveis deste mineral, considerando as correlações entre os níveis de fósforo consumido e de fósforo no plasma; na saliva; no conteúdo ruminal; nas fezes; e na urina. Foram utilizados 18 cordeiros com 5 meses de idade e $27 \mathrm{~kg}$ de peso vivo. O experimento foi dividido em dois períodos de cinco semanas, cada um com nove animais. Os animais foram mantidos individualmente em gaiolas para estudo de metabolismo e receberam uma dieta basal constituída de feno de capim coast-cross (Cynodon dactylon, L. Pers.) e concentrado com diferentes níveis de fósforo (1,9; 2,6; 3,3 g/dia) provenientes de fosfato bicálcico. No 15o dia, iniciaram-se as coletas de saliva, plasma, conteúdo ruminal, fezes e urina para as análises de fósforo inorgânico. Observaram-se correlações positivas entre o aumento do consumo de fósforo e os teores desse mineral no plasma $(r=0,64)$, na saliva $(r=0,86)$, no conteúdo ruminal $(r=0,82)$, nas fezes $(r=0,92)$ e na urina $(r=0,37)$, comprovando que, quando absorvido, o fósforo é distribuído no corpo pelo plasma. Após a saturação no organismo, o excesso é secretado via saliva, segue para o rúmen e, ao chegar ao intestino, é reabsorvido em menor quantidade e excretado via fezes. À medida que se aumentaram os níveis de fósforo na dieta, verificou-se aumento expressivo dos teores desse mineral na saliva, no plasma, no conteúdo ruminal e nas fezes.

Palavras-chave: cordeiros Santa Inês, níveis de fósforo, nutrição animal, ruminantes

\section{Dynamics of phosphorus in the body of sheep fed different levels of this mineral in the diets}

\begin{abstract}
The dynamics of phosphorus in the body of Santa Ines lambs fed diets with different levels of this mineral was evaluated, considering the correlations among the levels of phosphorus consumed, in plasma; in saliva, in rumen contents, in feces, and in urine. Eighteen lambs were used with five mo old and $27 \mathrm{~kg}$ BW. The experiment was divided into two periods of five weeks, each with nine animals. The animals were individually kept in cages of metabolism study and were fed a basal diet consists of coast-cross grass (Cynodon dactylon, L. Pers.) hay and concentrate with different phosphorus levels (1.9, 2.6, $3.3 \mathrm{~g} /$ day) from dicalcium phosphate. On the 15th day, it was initiated the collection of saliva, plasma, rumen contents, feces and urine for the analysis of inorganic phosphorus. Positive correlations were observed among the increase of phosphorus intake and the levels of this mineral in plasma, saliva, rumen contents, feces and urine, indicating that, when absorbed, the phosphorus is distributed throughout the body by the plasma. After saturation in the body, the excess is secreted through saliva, following for the rumen, and reaching the intestine; it is reabsorbed into smaller quantity and excreted via feces. As phosphorus levels increased in the diet, there was significant increase of these mineral levels in saliva, plasma, rumen contents and feces.
\end{abstract}

Key Words: animal nutrition, phosphorus levels, ruminants, Santa Inês lambs

\section{Introdução}

No metabolismo animal, o fósforo (P) é um mineral multifuncional e com importantes funções fisiológicas e bioquímicas
(González \& Silva, 2003) eimportância significativana atividade dos microrganismos do rúmen (Breves \& Schroder, 1991). Em virtude dessas importantes funções, tem sido um dos nutrientes mais pesquisados nas últimas décadas (Bravo et al., 2003). 
As glândulas salivares determinam aproximadamente $80 \%$ da secreção endógena de fósforo no trato gastrintestinal (AFRC, 1991) e tem importante função na manutenção da homeostase de fósforo em ruminantes (Tomas \& Somers, 1974). Os valores de fósforo na saliva de ovinos podem variar de acordo com as concentrações desse mineral no plasma, diretamente correlacionadas ao conteúdo da dieta (Ternouth, 1989). O aumento dos níveis de fósforo na dieta também reflete positivamente nos níveis desse mineral na saliva e no rúmen (Ternouth, 1990).

As fezes são a principal rota de excreção de fósforo em ruminantes. Existe relação linear positiva entre o fósforo consumido e o fósforo total excretado (Bravo et al., 2003). $\mathrm{O}$ excesso de desse mineral excretado pelas fezes pode contaminar os meios aquáticos pela lixiviação, uma vez que é um dos nutrientes mais poluentes do ambiente, principalmente em criações intensivas e limitadas a pequenos espaços (Tamminga, 2003). A redução da excreção de nutrientes inicia-se pela formulação da dieta (Cast, 2002).

A homeostase de fósforo em ruminantes é determinada basicamente pela secreção salivar de fósforo e pela excreção do excesso pelas fezes. As perdas fecais desse mineral podem variar de acordo com a quantidade ingerida, com a qualidade da dieta e com a individualidade animal (Tomas \& Somers, 1974; Vitti, 2000).

O entendimento da cinética dos fluxos de fósforo nos compartimentos, como trato digestivo, saliva, sangue, conteúdo ruminal, fezes e urina possibilita conhecer melhor o fluxo desse mineral no organismo e reduzir as quantidades fornecidas. Wu et al. (2000) e Wu (2003) sugeriram que a redução de $20 \%$ do fósforo dietético não afeta o desempenho dos animais e reduz 25 a $30 \%$ do fósforo eliminado pelas fezes.

Avaliou-se neste trabalho a dinâmica do fósforo no organismo e suas concentrações no plasma, na saliva, no conteúdo ruminal, nas fezes e na urina de ovinos Santa Inês alimentados com dietas com diversos níveis desse mineral.

\section{Material e Métodos}

O trabalho foi realizado no Departamento de Zootecnia da Universidade Federal de Lavras, em Lavras, Minas Gerais. Foram utilizados 18 cordeiros da raça Santa Inês, machos não-castrados, com 27,18 $\pm 1,56 \mathrm{~kg}$ e com 5 meses de idade, alojados em gaiolas para estudos de metabolismo.

O ensaio foi dividido em dois períodos de cinco semanas: as duas primeiras semanas corresponderam ao período pré-experimental e as outras três ao período de coletas. As coletas foram realizadas durante quatro dias consecutivos por semana, com três dias de intervalo antes da coleta seguinte. Em cada período, foram utilizados nove animais.

O experimento foi conduzido em delineamento de blocos casualizados, em esquema de parcelas subdivididas. Os blocos foram formados considerando o peso vivo como critério de blocagem. Cada período foi constituído de três blocos de três animais para um total de seis repetições. Os tratamentos (níveis de fósforo) corresponderam às parcelas e a semana experimental às subparcelas. A semana foi considerada medida repetida no tempo. Cada animal foi considerado uma unidade experimental.

As dietas, isocalóricas e isoprotéicas, diferiram apenas quanto aos níveis de fósforo e foram balanceadas utilizando-se as equações do AFRC (1993). A ração foi balanceada para possibilitar rápido crescimento dos animais com consumo à vontade. Os níveis de fósforo totais das dietas foram: $1,9 \mathrm{~g} / \mathrm{dia}-25 \%$ menos que a porcentagem total de fósforo recomendada pelo NRC (1985); 2,6 g/dia - nível recomendado pelo $\mathrm{NRC}$ (1985); e 3,3 g/dia - $25 \%$ a mais que o recomendado pelo NRC (1985).

As dietas experimentais (Tabela 1 ) foram compostas de $600 \mathrm{~g}$ de feno de coast-cross (Cynodon dactylon, L. Pers.) picado, $40 \mathrm{~g}$ de farelo de soja, $375 \mathrm{~g}$ milho moído, $300 \mathrm{~g}$ de polpa cítrica, $7 \mathrm{~g}$ de uréia, $10 \mathrm{~g}$ de mistura mineral e $500 \mathrm{UI} /$ animal/dia de vitamina $\mathrm{D}$. A relação cálcio:fósforo em todas

Tabela 1 - Composição das dietas experimentais (\% MS)

\begin{tabular}{|c|c|c|c|}
\hline \multirow[t]{2}{*}{ Ingrediente } & \multicolumn{3}{|c|}{ Nível de fósforo (g/dia) } \\
\hline & 1,9 & 2,6 & 3,3 \\
\hline Feno & 44,98 & 44,61 & 44,17 \\
\hline Milho & 28,12 & 27,88 & 27,61 \\
\hline Polpa cítrica & 22,62 & 22,43 & 22,21 \\
\hline Farelo de soja & 3,07 & 3,04 & 3,01 \\
\hline Uréia & 0,60 & 0,60 & 0,60 \\
\hline Calcário calcítico & 0,00 & 0,46 & 0,98 \\
\hline Fosfato bicálcico & 0,00 & 0,38 & 0,82 \\
\hline Mistural mineral $^{1}$ & 0,61 & 0,60 & 0,60 \\
\hline Total & 100,00 & 100,00 & 100,00 \\
\hline \multicolumn{4}{|l|}{ Composição nutricional } \\
\hline Matéria seca (\%) & 86,31 & 86,41 & 86,51 \\
\hline $\begin{array}{l}\text { Nutrientes digestíveis } \\
\text { totais }(\%)^{*}\end{array}$ & 63,60 & 63,01 & 62,42 \\
\hline Proteína bruta (\%) & 10,83 & 10,73 & 10,62 \\
\hline Extrato etéreo $(\%)$ & 3,40 & 3,36 & 3,33 \\
\hline $\begin{array}{l}\text { Fibra em detergente } \\
\text { neutro }(\%)\end{array}$ & 46,09 & 45,72 & 45,24 \\
\hline Cinzas $(\%)$ & 6,28 & 7,12 & 8,01 \\
\hline Cálcio (\%) & 0,68 & 0,92 & 1,18 \\
\hline Fósforo $(\%)$ & 0,18 & 0,25 & 0,33 \\
\hline
\end{tabular}

${ }^{1}$ CuSO4 - 0,09 g; CoSO4 - 0,0008 g; MnSO4 - 0,148 g; KI - 0,009 g; ZnSO4 - 0,32 g; FeSO4 - 0,457 g; NaCl - 3,4 g; MgO - 1,61 g; S - 4,0 g; $\mathrm{Na}_{2} \mathrm{SeO}_{3}-0,001$

* Estimado pelo NRC (1985) 
as dietas foi mantida igual, por meio da utilização de calcário calcítico. As quantidades da dieta oferecida permitiram sobras de $20 \%$ do total oferecido.

As fezes coletadas e pesadas diariamente foram amostradas (10\% do total)e, posteriormente, homogeneizadas, pesadas e mantidas em estufa a $55^{\circ} \mathrm{C}$ com ventilação forçada por 72 horas. As amostras foram moídas em peneira com crivos de $1 \mathrm{~mm}$ e colocadas em estufa a $105^{\circ} \mathrm{C}$ por 12 horas para determinação do teor de matéria seca (MS). Para determinação das concentrações de fósforo, foram utilizados $5 \mathrm{~g}$ de cada amostra, que foram colocados em cadinhos de porcelana, incinerados a $550^{\circ} \mathrm{C}$ e dissolvidos com $\mathrm{HCl}$ concentrado. Em seguida, o conteúdo dos cadinhos foi filtrado e transferido para balões volumétricos de $100 \mathrm{~mL}$, cujos volumes foram completados com água destilada.

Os mesmos procedimentos adotados nas análises das fezes foram utilizados na análise dos teores de fósforo das dietas e das sobras. A determinação de fósforo inorgânico nos alimentos, nas sobras e nas fezes foi feita por colorimetria utilizando-se o método vanadato-molibdato (Sarruge \& Haag, 1974)

Amostras de sangue foram coletadas na veia jugular de cada animal em tubos contendo heparina. Em seguida, foram centrifugadas (3.000 rpm por 15 minutos) para separação do plasma, que foi mantido congelado até a análise. Amostras de saliva também foram coletadas com auxílio de uma pinça e uma esponja plástica, diretamente na boca dos animais. O conteúdo ruminal foi amostrado com auxílio de sonda esofagiana de $1 \mathrm{~cm}$ de diâmetro. $O$ conteúdo ruminal foi aspirado com uma seringa de $50 \mathrm{~mL}$, filtrado com gaze, diluído 1:10 com água destilada e centrifugado a 3.000 rpm por 15 minutos. A urina total foi coletada em recipientes plásticos colocados sob as gaiolas.

Compuseram-se amostras compostas de plasma, saliva, conteúdo ruminal e urina para análises e determinação das concentrações de fósforo nos quatros dias de cada semana para cada animal. De cada amostra de plasma, saliva, con- teúdo ruminal e urina, retirou-se $1 / 2 \mathrm{~mL}$, que foi misturado a 4,5 mL de ácido tricloroacético a $10 \%$ para precipitação da proteína. Dez minutos depois, o material foi centrifugado a $3.000 \mathrm{rpm}$ por 15 minutos e as determinações de fósforo inorgânico foram feitas por colorimetria, conforme metodologia descrita por Fiske \& Subbarrow (1925).

Para os parâmetros avaliados, foram feitas correlações de Pearson utilizando-se o programa SAEG, Sistema de Análises Estatísticas e Genéticas (Euclydes, 1983).

\section{Resultados e Discussão}

O peso vivo e o metabolismo de fósforo dos animais não foram influenciados pela interação nível de fósforo $x$ semana de coletas nem diferiram estatisticamente entre as semanas de avaliação (Tabela 2).

A ingestão de MS não esteve relacionada à ingestão de fósforo, o que confirma os resultados obtidos por Portilho et al. (2006), que observaram consumos constantes de MS quando forneceram dietas com diferentes níveis de fósforo em ovinos Santa Inês. Quando há severa deficiência de fósforo na dieta, o consumo de MS pode ser reduzido, como resultado da redução na atividade microbiana no rúmen, responsável pela degradação do alimento (Ternouth, 1990). Esta influência não foi observada neste trabalho, uma vez que a deficiência de fósforo na dieta não foi expressiva - apenas $25 \%$ abaixo dos níveis recomendados pelo NRC (1985).

Autores como Abdelrahaman (1998) e Antunes (2006) observaram correlações positivas entre níveis de ingestão de fósforo e níveis séricos de fósforo. Neste trabalho, também houve correlação positiva $(\mathrm{r}=0,64 ; \mathrm{P}<0,01$; Figura 1$)$ entre o fósforo consumido e o fósforo no plasma. Outros autores (Silva Filho, 1995; Portilho, 2003) contestam que a homeostase do fósforo é mantida por mecanismos que se ajustam às condições fisiológicas do animal, o que tem provocado contradições quando se relacionam fósforo no

Tabela 2 - Peso vivo e metabolismo de fósforo em cordeiros alimentados com dietas contendo diferentes níveis desse mineral (médias ${ }^{1} \pm$ desvio-padrão)

\begin{tabular}{|c|c|c|c|c|c|c|c|}
\hline & $\mathrm{P}-\mathrm{cons}$ & $\mathrm{P}-$ plas & $\mathrm{P}-\mathrm{sal}$ & $P-f e z$ & CMS & $P-$ crum & $P-$ uri \\
\hline $\mathrm{P}-\mathrm{cons}$ & 1 & $0,64 * *$ & $0,86^{* *}$ & $0,92 * *$ & $-0,11^{\mathrm{NS}}$ & $0,82 * *$ & $0,37 * *$ \\
\hline $\mathrm{P}-$ plas & & 1 & $0,73 * *$ & $0,56 * *$ & $0,25 *$ & $0,67 * *$ & $0,39 * *$ \\
\hline $\mathrm{P}-\mathrm{sal}$ & & & 1 & $0,79 * *$ & $0,00^{\mathrm{NS}}$ & $0,87 * *$ & $0,34 * *$ \\
\hline$P-f e z$ & & & & 1 & $-0,04^{\mathrm{NS}}$ & $0,76 * *$ & $0,32 * *$ \\
\hline CMS & & & & & 1 & $-0,17^{\mathrm{NS}}$ & $-0,01^{\mathrm{NS}}$ \\
\hline $\mathrm{P}-\mathrm{crum}$ & & & & & & 1 & $0,44 * *$ \\
\hline $\mathrm{P}-$ uri & & & & & & & 1 \\
\hline
\end{tabular}

P-cons: fósforo consumido; P-plas: fósforo no plasma; P-sal: fósforo na saliva; P-fez: fósforo nas fezes; CMS: consumo de matéria seca; P-crum: fósforo no conteúdo ruminal; P-uri: fósforo na urina.

${ }^{* *}=(\mathrm{P}<0,01) ;{ }^{*}=(\mathrm{P}<0,05) ; \mathrm{NS}=$ não-significativo 


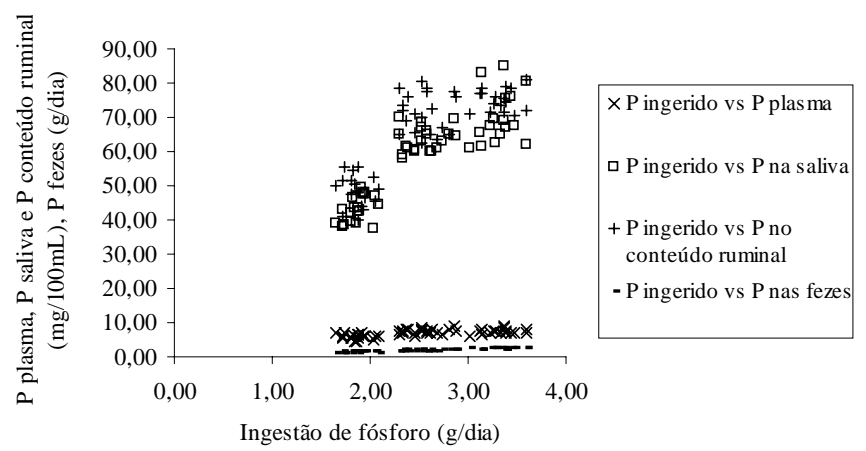

Figura 1 - Correlações entre o fósforo ingerido e o fósforo no plasma, na saliva, no conteúdo ruminal e nas fezes.

plasma e fósforo consumido. Os níveis de fósforo no plasma podem também se alterar com a idade, o comportamento do animal e o estresse durante a coleta e manipulação inadequada das amostras (McDowell et al., 1986).

O nível de fósforo no plasma esteve correlacionado ao teor desse mineral na saliva $(r=0,73 ; \mathrm{P}<0,01$; Figura 2$)$, o que reforça relatos de Louvandini \& Vitti (1994), $\mathrm{r}=0,54$ e $\mathrm{P}<0,01$, e Portilho (2003), $\mathrm{r}=0,65$ e $\mathrm{P}<0,05$, que observaram correlações positivas entre os níveis de fósforo no plasma e na saliva. Esses resultados se devem ao ciclo do fósforo, que, ao ser absorvido, é distribuído no organismo pelo plasma e retorna ao rúmen pela secreção salivar, que desempenha importantes funções, como o fornecimento de fósforo para as bactérias do rúmen, a manutenção do $\mathrm{pH}$ ruminal por meio de sua ação tampão e a excreção do excesso de fósforo absorvido.

Observou-se também alta correlação entre o fósforo ingerido e suas concentrações na saliva $(r=0,86 ; \mathrm{P}<0,01$; Figura 1), logo, a alta ingestão de fósforo promove maior absorção (Rosol \& Capen, 1997) e aumento nas concentrações plasmáticas, o que resulta em maiores concentrações desse mineral na saliva e contribui para sua homeostase no organismo (Tomas \& Somers, 1974).

Houve correlação positiva entre fósforo ingerido e suas concentrações no conteúdo ruminal $(\mathrm{r}=0,82 ; \mathrm{P}<0,01$; Figura 1$)$, que aumentaram de acordo com a ingestão de fósforo. Observou-se também correlação positiva entre fósforo no conteúdo ruminal e fósforo na saliva $(\mathrm{r}=0,87 ; \mathrm{P}<0,01$; Figura 3$)$ e entre o fósforo no conteúdo ruminal e fósforo no plasma $(\mathrm{r}=0,67$; $\mathrm{P}<0,01$; Tabela 3), o que confirma os resultados descritos por Tomas et al. (1967) e Vitti et al. (1988).

Essas correlações podem ser atribuídas ao fato de os ruminantes secretarem dentro do rúmen grandes quantidades de saliva, que supre grande parte das necessidades de fósforo dos microrganismos do rúmen (Scott \& Buchan, 1988). A saliva, além de ser importante na manutenção da

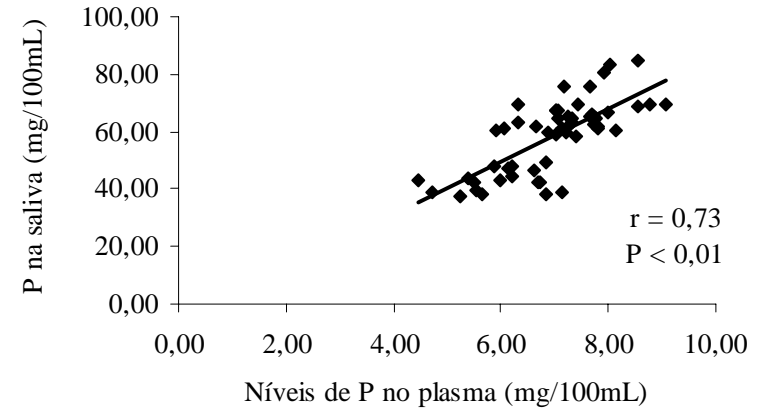

Figura 2 - Correlação entre o fósforo no plasma e o fósforo na saliva.

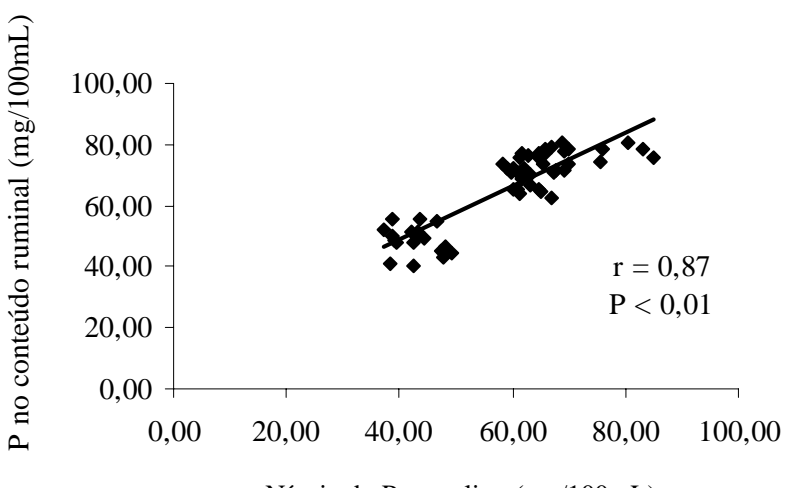

Figura 3 - Correlação entre fósforo no conteúdo ruminal e fósforo na saliva.

homeostase de fósforo em ruminantes (Tomas \& Somers, 1974), representa $80 \%$ da secreção endógena desse mineral (AFRC, 1991), que perfaz boa parte do total de fósforo excretado nas fezes e é regulada principalmente pela concentração do fósforo no plasma (Scott et al., 1985; Ternouth, 1989).

A alta correlação positiva entre o conteúdo de fósforo fecal e o de fósforo consumido $(\mathrm{r}=0,92 ; \mathrm{P}<0,01$; Figura 1$)$ tem sido observada por outros pesquisadores, que classificam as fezes como principal rota de excreção de fósforo em ruminantes. Verifica-se, portanto, relação positiva entre o fósforo consumido e o fósforo total excretado (Bueno \& Vitti, 1999; Bravo et al., 2003; Antunes, 2006).

Portilho (2003) também obteve dados semelhantes em experimentos com cordeiros. Os maiores níveis de fósforo nas fezes, segundo esse autor, corresponderam aos níveis mais elevados de fósforo na dieta. As perdas de fósforo pelas fezes podem predizer a ingestão ou a absorção do mineral, pois a homeostase desse mineral em ruminantes atinge quase totalmente o seu equilíbrio no trato gastrointestinal, em virtude do controle da secreção e reabsorção do fósforo da saliva (Clark et al., 1973). Neste trabalho, essa 
tendência também foi observada e reforçada pelas correlações entre fósforo nas fezes e o conteúdo ruminal $(\mathrm{r}=0,76$; $\mathrm{P}<0,01$; Tabela 3); entre o fósforo nas fezes e fósforo salivar ( $\mathrm{r}=0,79 ; \mathrm{P}<0,01 ;$ Tabela 3$)$; e entre o fósforo nas fezes e fósforo no plasma $(\mathrm{r}=0,56 ; \mathrm{P}<0,01$; Tabela 3$)$. O fósforo, quando absorvido, é distribuído no organismo pelo plasma e, após saturação no corpo, seu excesso é secretado via saliva e segue para o rúmen. Ao chegar no intestino, a reabsorção diminui aumentando a excreção via fezes (Lobão et al., 1974).

Verificou-se baixa correlação linear $(r=0,37, P<0,05$; Tabela 3), embora positiva, entre o fósforo ingerido e o excretado na urina. Segundo Challa et al. (1989), as perdas urinárias de fósforo normalmente não estão relacionadas à ingestão de fósforo, mas estão associadas à eficiência de absorção. Esses autores ressaltam que as glândulas salivares controlam de forma eficiente o fósforo plasmático, fato verificado por Portilho (2003), que observou correlação positiva entre fósforo no plasma e na urina $(\mathrm{r}=0,81 ; \mathrm{P}<0,01)$. Neste trabalho, no entanto, a correlação entre esses dois parâmetros foi baixa $(\mathrm{r}=0,39 ; \mathrm{P}<0,05$; Tabela 3$)$.

\section{Conclusões}

Os teores de fósforo na saliva, no plasma, no conteúdo ruminal e nas fezes aumentam de acordo com o conteúdo desse mineral na dieta. Menores ingestões de fósforo, correspondentes a $25 \%$ menos que o recomendado pelo NRC (1985), são suficientes para manter os níveis de fósforo na saliva, no plasma, no conteúdo ruminal, nas fezes e na urina nas faixas consideradas normais, portanto, a exigência de fósforo em ovinos Santa Inês pode ser menor que a preconizada pelo NRC (1985).

\section{Literatura Citada}

ALBDELRAHMAN, M.M.; KINCAID, R.L.; ELZUBEIR, E.A. Mineral deficiencies in grazing dairy cattle in Kordofan and Darfur regions in Western Sudan. Tropical Animal Health Production, v.30, p.123-135. 1998.

AGRICULTURAL AND FOOD RESEARCH COUNCIL - AFRC Technical Committee on Responses to Nutrients. A reappraisal of the calcium and phosphorus requirements of sheep and cattle. Nutrition Abstracts and Reviews Series, v.61, n.9, p.573$612,1991$.

AGRICULTURAL AND FOOD RESEARCH COUNCIL - AFRC. Energy and protein requirements of ruminants. Wallingford: CAB International, 1993. 159p.

ANTUNES, D.A. Phosphorus deficiency diagnosis in sheep using labeled phosphorus uptake by erythrocytes. Pesquisa Agropecuária Brasileira, v.41, n.2, p.339-346, 2006.

BRAVO, D.; SUVANT, D.; BOGAERT, C. et al. A bibliographic database for quantitative analysis of phosphorus flow in ruminants. Reproduction Nutrition Development, v.43, p.251$269,2003$.
BREVES, G.; SCHRÖDER, B. Comparative aspects of gastrointestinal phosphorus metabolism. Nutrition Research Reviews, v.4, p.125-140, 1991 .

BUENO, M.S.; VITTI, D.M.S.S. Níveis de fósforo para caprinos: Perda endógena fecal exigência líquida para mantença. Pesquisa Agropecuária Brasileira, v.34, n.4, p.675-681, 1999.

COUNCIL FOR AGRICULTURE SCIENCE AND TECHNOLOGY - CAST. Animal diet modification to decrease the potential for nitrogen and phosphorus pollution. Ames: 2002. 16p. (Publication, 21).

CHALLA, J.; BRAITHWAITE, G.D.; DHANOA, M.S. Phosphorus homeostasis in growing calves. Journal Agricultural Science, v.112, n.3, p.217-226, 1989.

CLARK, R.C.; BUDTZ-OLZEN, O.E.; CROSS, R.B. The importance of the salivary glands in the maintence of phosphorus homeostasis in the sheep. Australian Journal of Agricultural Research, v.24, n.6, p.913-919, 1973.

EUCLYDES, R.F. Sistemas para análises estatísticas - SAEG. Viçosa, MG: Universidade Federal de Viçosa, 1983. 59p.

FISKE, C.H.; SUBBARROW, Y. The colorimetric determination of phosphorus. Journal of Biological Chemistry, v.66, n.2, p.375-400, 1925.

GONZÁLEZ, F.H.D.; SILVA, S.C. Introdução à bioquímica clínica animal. Porto Alegre: Universidade Federal do Rio Grande do Sul, 2003. 360p.

LOBÃO, A.O.; CROCOMO, O.J. Retenção de fósforo radotivo $\left.{ }^{32} \mathrm{P}\right)$ em tecidos de ovinos. Boletim de Indústria Animal, v.31, n.2, p.261-291, 1974.

LOUVANDINI, H.; VITTI, D.M.S.S. Perda endógena de fósforo em ovinos com diferentes níveis do elemento na dieta. Pesquisa Agropecuária Brasileira, v.29, n.1, p.145-149, 1994.

MCDOWELL, L.R.; CONRAD, J.H.; LOOSLI, F.K. Mineral imbalances and their diagnosis in ruminant. In: INTERNATIONAL ATOMIC ENERGY AGENCY (Vienna, Áustria). Nuclear and related techniques in animal production and health. Vienna: 1986. p.521-534.

NATIONAL RESEARCH COUNCIL - NRC. Nutrient requirements of domestic animals: nutrient requirements of sheep. Washington, D.C.: National Academy of Science, 1985. 99p.

PORTILHO, F.P. Exigência mínima de fósforo para cordeiros da raça Santa Inês. Brasília: Universidade de Brasília, 2003. 68p. Dissertação (Mestrado em Ciências Agrárias) - Universidade de Brasília, 2003.

PORTILHO, F.P.; VITTI, D.M.S.S.; ABDALLA, A.L. et al. Minimum phosphorus requirement for Santa Inês lambs reared under tropical conditions. Small ruminant Research, v.63, p.170-176. 2006.

ROSOL, T.J.; CAPEN, C.C. Calcium-regulating hormones and diseases of abnormal mineral (calcium, phosphorus, magnesium) metabolism. In: KANEKO, J.J (Ed.) Clinical biochemistry of domestics animals. 5.ed. New York: Academic Press, 1997. $932 p$.

SARRUGE, J.R.; HAAG, H.P. Análises químicas em plantas. Piracicaba: ESALQ/USP, 1974. 56p.

SILVA FILHO, J.C. Avaliação da incorporação de 32P pelos eritrócitos como método para diagnóstico de deficiência subclínica de fósforo em ruminantes. Piracicaba: Universidade de São Paulo, 1995. 62p. Tese (Doutorado em Energia Nuclear na Agricultura) - Universidade de São Paulo, 1995.

SCOTT, D.; WHITELAW, F.G.; BUCHAN, W. et al. The effect of variation in phosphorus intake on salivary phosphorus secretion, net intestinal phosphorus absorption and faecal endogenous excretion in sheep. Journal of Agricultural Science, v.105, n.3, p.271-277, 1985.

SCOTT, D.; BUCHAN, W. The effects of feeding pelleted diets made from either coarsely or finely ground hay on phosphorus balance and on the partition of phosphorus excretion between urine and faeces in the sheep. Quartely Journal of Experimental Physiology, v.73, n.3, p.315-322, 1988. 
TAMMINGA, S. Pollution due to nutrient losses and its control in European animal production. Livestock Production Science, v.84, n.2, p.101-111, 2003

TERNOUTH, J.H. Endogenous losses of phosphorus by sheep Journal of Agriculture Science, v.113, n.3, p.291-297, 1989.

TERNOUTH, J.H. Phosphorus and beef production in Northern Australia. 3. Phosphorus in cattle a review. Tropical Grassland, v.24, n.3, p.159-169, 1990

TOMAS, F.M.; MOIR, R.J.; SOMERS, M. Phosphorus turnover in sheep. Australian Journal of Agricultural Research, v.18, n.4, p.635-645, 1967.

TOMAS, F.M. SOMERS, M. Phosphorus homeostasis in sheep. I. Effect of ligation of parotid salivary ducts. Australian Journal of Agricultural Research, v.25, n.3, p.475-483, 1974.

VITTI, D.M.S.S.; ABDALLA, A.L.; SILVA FILHO, J.C. et al. Métodos para o diagnóstico da deficiência de fósforo em ruminantes. Pesquisa Agropecuária Brasileira, v.23, n.6, 645-51, 1988. VITTI, D.M.S.S.; KEBREAB, E.; ABDALLA, A.L. et al. kinetic model of phosphorus metabolism in growing goats. Journal of Animal Science, v.78, n.10, p.2706-2716, 2000.

WU, Z. People still are feeding too much phosphorus. Hoard's Dairyamn, v.148, n.11, p.210, 2003.

WU, Z.; SATTER L.D.; SOJO, R. Milk production, reproductive performance, and fecal excretion of phosphorus by dairy cows fed three amounts of phosphorus. Journal of Dairy Science, v.83, p.1028-1041, 2000. 